\title{
On $p$-superlinear equations with a nonhomogeneous differential operator
}

\author{
Sergiu Aizicovici, Nikolaos S. Papageorgiou and Vasile Staicu
}

\begin{abstract}
We consider a nonlinear elliptic equation driven by a nonhomogeneous differential operator and with a $(p-1)$-superlinear Carathéodory reaction. Our formulation incorporates as a special case equations monitored by the $p$-Laplacian. Using variational methods coupled with truncation techniques and comparison principles, we show that the problem has at least five nontrivial smooth solutions.
\end{abstract}

Mathematics Subject Classification (2000). 35J20, 35J60.

Keywords. Nonhomogeneous differential operator, Superlinear reaction, Nonlinear regularity, Nonlinear maximum principle, Comparison results, Local minimizer.

\section{Introduction}

In this paper we study the following nonlinear Dirichlet problem

$$
\left\{\begin{array}{l}
-\operatorname{div} a(z, D u(z))=f(z, u(z)) \quad \text { in } \Omega \\
\left.u\right|_{\partial \Omega}=0
\end{array}\right.
$$

where $\Omega \subset \mathbb{R}^{N}$ is a bounded domain with a $C^{2}$-boundary $\partial \Omega$, $a: \bar{\Omega} \times \mathbb{R}^{N} \rightarrow \mathbb{R}^{N}$ is continuous, of class $C^{1}$ on $\bar{\Omega} \times\left(\mathbb{R}^{N} \backslash\{0\}\right)$ and such that for all $z \in \bar{\Omega}, a(z,$.$) is$ strictly monotone. The precise conditions on $a(z, y)$ are formulated in hypotheses H(a) (see Sect. 2) and they incorporate as a special case the $p$-Laplacian differential operator. Also, $f(z, x)$ is a Carathéodory reaction (i.e., for all $x \in \mathbb{R} z \rightarrow f(z, x)$ is measurable and for almost all $z \in \Omega, x \rightarrow f(z, x)$ is continuous) and $x \rightarrow f(z, x)$ exhibits a $(p-1)$-superlinear growth near $\pm \infty, 1<p<\infty$, but without satisfying the usual in such case AmbrosettiRabinowitz condition (AR-condition for short). Our aim in this paper is to prove a multiplicity result for such problems.

Dedicated to Arrigo Cellina on the occasion of his 70th birthday. 
Recall that a Carathéodory function $f(z, x)$ satisfies the AR-condition, if there exist constants $q>p$ and $M>0$ such that

$$
0<q F(z, x) \leq f(z, x) x \quad \text { for a.e. } z \in \Omega \text {, all }|x| \geq M,
$$

where $F(z, x)=.\int_{0}^{x} f(z, s) d s$. Integrating (1.2), we obtain the weaker condition

$$
\widehat{c}|x|^{q} \leq F(z, x) \text { for a.e. } z \in \Omega \text {, all }|x| \geq M \text {, with } \widehat{c}>0 .
$$

From (1.3) follows the much weaker condition

$$
\lim _{x \rightarrow \pm \infty} \frac{F(z, x)}{|x|^{p}}= \pm \infty \quad \text { uniformly for a.e. } z \in \Omega .
$$

Evidently (1.4) implies that the primitive $F(z,$.$) is p$-superlinear near $\pm \infty$.

In this paper we employ (1.4) together with an asymptotic condition on the function

$$
x \rightarrow \sigma(z, x):=f(z, x)-p F(z, x) .
$$

This condition allows us to incorporate in our framework of analysis $(p-1)$ superlinear reaction terms which exhibit slower growth near $\pm \infty$ and whose primitives do not satisfy the AR-condition (1.2) or even the more general condition (1.3).

The AR-condition ensures in a straightforward way that the energy functional of the problem satisfies the PS-condition (cf. Sect. 2). Recently, there have been efforts to drop it. In this direction we mention the works of Jeanjean [20], Myagaki and Souto [27], Schechter and Zou [31] (semilinear problems) and Fang and Liu [13], Li and Yang [25], Su [32] (problems driven by the $p$-Laplacian).

Our superlinear condition here is different from the ones used in the aforementioned works.

Problems driven by nonhomogeneous differential operators were investigated by Dabuleanu and Radulescu [9], De Napoli and Mariani [11], Duc and $\mathrm{Vu}$ [12], $\mathrm{Hu}$ and Papageorgiou [18], Kourogenis and Papageorgiou [21], Kristaly et al. [22], Kyritsi et al. [28] and Papageorgiou et al. [29].

Multiplicity results were proved in [11] (under symmetry conditions on the reaction term) and in $[22,23,28,29]$ for certain classes of parametric equations. The $p$-superlinear case is considered only in [28] under a different hypothesis on the reaction. All these works produce at most three nontrivial solutions.

Finally we mention the recent papers of the authors $[1,3,4]$, on "superlinear" Neumann problems driven by the $p$-Laplacian.

\section{Mathematical background: auxiliary results}

Our approach is variational, based on the critical point theory. We also use suitable truncation techniques and comparison results. Let use recall some basic definitions and facts from critical point theory, which we will need in the sequel. 
So, let $(X,\|\|$.$) be a Banach space and X^{*}$ be its topological dual. By $\langle.,$.$\rangle we denote the duality brackets for the pair \left(X^{*}, X\right)$. Let $\varphi \in C^{1}(X)$. A real number $c$ is said to be a critical value of $\varphi$ if there exists $x^{*} \in X$ such that $\varphi^{\prime}\left(x^{*}\right)=0$ and $\varphi\left(x^{*}\right)=c$.

We say that $\varphi$ satisfies the Cerami condition (the C-condition for short), if if the following holds:

"every sequence $\left\{x_{n}\right\}_{n \geq 1} \subseteq X$ such that

$\left\{\varphi\left(x_{n}\right)\right\}_{n \geq 1}$ is bounded in $\mathbb{R}$ and $\left(1+\left\|x_{n}\right\|\right) \varphi^{\prime}\left(x_{n}\right) \rightarrow 0$ in $X^{*}$ as $n \rightarrow \infty$,

admits a strongly convergent subsequence."

This condition is more general than the usual in critical point theory Palais-Smale condition (the PS-condition for short). However, it can be shown (see, for example, Gasinski and Papageorgiou [16]) that the deformation theorem and consequently the minimax theory of critical values, remain valid if the PS-condition is replaced by the weaker C-condition.

The next result is known in the literature as the "mountain pass theorem".

Theorem 1. If $\varphi \in C^{1}(X)$ and satisfies the $C$-condition, $x_{0}, x_{1} \in X$ and $r>0$ satisfy $\left\|x_{1}-x_{0}\right\|>r$ and

$$
\begin{aligned}
& \max \left\{\varphi\left(x_{0}\right), \varphi\left(x_{1}\right)\right\}<\inf \left\{\varphi(x):\left\|x-x_{0}\right\|=r\right\}=: \eta_{r}, \\
& c:=\inf _{\gamma \in \Gamma} \max _{t \in[0,1]} \varphi(\gamma(t)) \text { where } \\
& \Gamma=\left\{\gamma \in C([0,1], X): \gamma(0)=x_{0}, \gamma(1)=x_{1}\right\}
\end{aligned}
$$

then $c \geq \eta_{r}$ and $c$ is a critical value of $\varphi$.

In what follows, we will use the same symbol, $\|$.$\| , to indicate the norms$ in $\mathbb{R}^{N}$ and $W_{0}^{1, p}(\Omega)$, whenever there is no danger of confusion. The inner product in $\mathbb{R}^{N}$ and the norm in $L^{p}(\Omega)\left(\right.$ or $\left.L^{p}\left(\Omega, \mathbb{R}^{N}\right)\right)$ will be denoted by $(., .)_{\mathbb{R}^{N}}$ and $\|\cdot\|_{p}$, respectively. Finally, $\stackrel{w}{\rightarrow}$ will designate weak convergence.

The hypotheses on the map $a(.,$.$) are the following$

$\mathbf{H}(\mathbf{a})$ : For all $(z, y) \in \bar{\Omega} \times \mathbb{R}^{N}$ one has $a(z, y)=h(z,\|y\|) y$ where $h: \bar{\Omega} \times$ $(0, \infty) \rightarrow \mathbb{R}$ is such that $h(z, t)>0$ for all $(z, t) \in \bar{\Omega} \times(0, \infty)$ and $\lim _{t \rightarrow 0^{+}} h(z, t) t=0 \forall z \in \bar{\Omega} ;$ moreover,

(i) $\quad a \in C\left(\bar{\Omega} \times \mathbb{R}^{N}, \mathbb{R}^{N}\right) \cap C^{1}\left(\bar{\Omega} \times\left(\mathbb{R}^{N} \backslash\{0\}\right), \mathbb{R}^{N}\right)$;

(ii) for every $z \in \bar{\Omega}$ and every $y \in \mathbb{R}^{N} \backslash\{0\}$ we have

$$
\left\|\nabla_{y} a(z, y)\right\| \leq C_{1}\|y\|^{p-2} \text { with } C_{1}>0 \text { and } 1<p<\infty \text {; }
$$

(iii) for every $z \in \bar{\Omega}$ and every $y \in \mathbb{R}^{N} \backslash\{0\}$ we have

$$
\left(\nabla_{y} a(z, y) \xi, \xi\right)_{\mathbb{R}^{N}} \geq C_{0}\|y\|^{p-2}\|\xi\|^{2} \quad \forall \xi \in \mathbb{R}^{N},
$$

for some $C_{0}>0$;

(iv) for every $\rho>0$ there exists $\alpha \in(0,1]$ such that for every $y \in$ $\mathbb{R}^{N} \backslash\{0\}$ with $\|y\| \leq \rho$ we have

$$
\left|a(z, y)-a\left(z^{\prime}, y\right)\right| \leq C_{2}\left\|z-z^{\prime}\right\|^{\alpha} \quad \forall z, z^{\prime} \in \bar{\Omega},
$$

for some $C_{2}>0$; 
(v) there exists a function $\beta \in L^{1}(\Omega)$ such that

$$
h(z, t) t^{2}-p \int_{0}^{t} h(z, s) s d s \leq \beta(z) \text { for a.e. } z \in \Omega, \text { all } t>0 .
$$

Remarks. Let $g(z, t)=h(z, t) t$ for all $z \in \bar{\Omega}$ and all $t \geq 0$. Hypotheses $\mathbf{H}(\mathbf{a})$ imply the following one-dimensional estimate

$$
C_{0} t^{p-2} \leq g_{t}^{\prime}(z, t) \leq C_{1} t^{p-2} \text { for all } z \in \bar{\Omega} \text { and all } t>0 .
$$

Note that

$$
(a(z, y), y)_{\mathbb{R}^{N}}=h(z,\|y\|)\|y\|^{2}
$$

and the primitive of $a$ with respect to $y$ is given by

$$
G(z, y)=\int_{0}^{\|y\|} h(z, s) s d s .
$$

Moreover, the convexity of $G(z,$.$) implies$

$$
(a(z, y), y)_{\mathbb{R}^{N}} \geq G(z, y) \quad \text { for all }(z, y) \in \bar{\Omega} \times \mathbb{R}^{N} .
$$

We mention that hypotheses $\mathbf{H}(\mathbf{a})$ are more general than the ones employed by Kyritsi et al. [23] and by Papageorgiou and Rocha [28]. Moreover, hypotheses $\mathbf{H}(\mathbf{a})$ imply that $G(z,$.$) is strictly convex, in contrast to the works$ of De Napoli and Mariani [11] and Kristaly et al. [22], where it is required that $G(z,$.$) is strongly convex. In the special case of the p$-Laplacian, this implies $p \geq 2$. obtain

Using $\mathbf{H}(\mathbf{a})$, (2.1) and the integral form of the mean value theorem, we

Lemma 1. If hypotheses $\mathbf{H}(\mathbf{a})$ hold, then

(a) for all $z \in \bar{\Omega}, y \rightarrow a(z, y)$ is maximal monotone and strictly monotone;

(b) for all $(z, y) \in \bar{\Omega} \times \mathbb{R}^{N},\|a(z, y)\| \leq \frac{C_{1}}{p-1}\|y\|^{p-1}$;

(c) for all $(z, y) \in \bar{\Omega} \times \mathbb{R}^{N},(a(z, y), y)_{\mathbb{R}^{N}} \geq \frac{C_{0}}{p-1}\|y\|^{p}$.

This lemma leads to the following growth estimates for the potential $G(z, y)$ :

Corollary 1. If hypotheses $\mathbf{H}(\mathbf{a})$ hold, then for all $(z, y) \in \bar{\Omega} \times \mathbb{R}^{N}$ we have

$$
\frac{C_{0}}{p(p-1)}\|y\|^{p} \leq G(z, y) \leq \frac{C_{1}}{p(p-1)}\|y\|^{p} .
$$

Example. The following maps satisfy hypotheses $\mathbf{H}(\mathbf{a})$. In all these examples $\theta \in C^{0, \alpha}(\bar{\Omega})(0<\alpha \leq 1)$ with $\theta(z)>0$ for all $z \in \bar{\Omega}$; specifically

(a) $a(z, y)=\theta(z)\|y\|^{p-2} y, 1<p<\infty$ (it corresponds to the weighted p-Laplacian);

(b) $\quad a(z, y)=\theta(z)\left[\|y\|^{p-2} y+\ln \left(1+\|y\|^{p-2}\right) y\right], p \geq 2$;

(c) $a(z, y)=\left\{\begin{array}{ll}\theta(z)\left[\|y\|^{p-2} y+\|y\|^{q-2} y\right] & \text { if }\|y\| \leq 1 \\ \theta(z)\left[\|y\|^{p-2} y+\frac{q-2}{\tau-2}\|y\|^{\tau-2} y-\frac{q-\tau}{\tau-2} y\right] & \text { if }\|y\|>1\end{array}\right.$ ， $2<\tau \leq p \leq q<\infty$ 
(d) $a(z, y)=\theta(z)\left[\|y\|^{p-2} y+C \frac{\|y\|^{p-2} y}{1+\|y\|^{p}}\right]$, with $C>0$ (it corresponds to the weighted sum of the $p$-Laplacian and a generalized mean curvature operator).

Let $V: W_{0}^{1, p}(\Omega) \rightarrow W^{-1, p^{\prime}}(\Omega)=W_{0}^{1, p}(\Omega)^{*}\left(\frac{1}{p}+\frac{1}{p^{\prime}}=1\right)$ be the nonlinear map defined by

$$
\langle V(u), y\rangle=\int_{\Omega}(a(z, D u), D y)_{\mathbb{R}^{N}} d z \quad \text { for all } u, y \in W_{0}^{1, p}(\Omega) .
$$

From Papageorgiou et al. [29] we have:

Proposition 1. If hypotheses $\mathbf{H}(\mathbf{a})$ hold, then the nonlinear map $V: W_{0}^{1, p}(\Omega) \rightarrow$ $W^{-1, p^{\prime}}(\Omega)$ defined by $(2.2)$ is maximal monotone, strictly monotone and of type $S_{+}$(i.e., if $u_{n} \stackrel{w}{\rightarrow} u$ in $W_{0}^{1, p}(\Omega)$ and

$$
\limsup _{n \rightarrow \infty}\left\langle V\left(u_{n}\right), u_{n}-u\right\rangle \leq 0
$$

then $u_{n} \rightarrow u$ in $W_{0}^{1, p}(\Omega)$.)

In the analysis of problem (1.1), in addition to the Sobolev space $W_{0}^{1, p}(\Omega)$, we will also use the ordered Banach space

$$
C_{0}^{1}(\bar{\Omega})=\left\{u \in C^{1}(\bar{\Omega}): u=0 \text { on } \partial \Omega\right\} .
$$

The positive cone of this space is

$$
\mathcal{C}_{+}=\left\{u \in C_{0}^{1}(\bar{\Omega}): u(z) \geq 0 \quad \text { for all } z \in \bar{\Omega}\right\} .
$$

This cone has a nonempty interior given by

$$
\text { int } \mathcal{C}_{+}=\left\{u \in \mathcal{C}_{+}: u(z)>0 \quad \text { for all } z \in \Omega \text { and } \frac{\partial u}{\partial n}<0 \text { on } \partial \Omega\right\}
$$

where $n($.$) is the outward unit normal on \partial \Omega$.

In what follows, by $\|\cdot\|_{C_{0}^{1}(\bar{\Omega})}$ we denote the norm of $C_{0}^{1}(\bar{\Omega})$. Recall that by Poincaré's inequality $\|u\|$ is equivalent to $\|D u\|_{p}, \forall u \in W^{1, p}(\Omega)$.

Let $f_{0}: \Omega \times \mathbb{R} \rightarrow \mathbb{R}$ be a Carathéodory function with subcritical growth in $x \in \mathbb{R}$, i.e.,

$$
\left|f_{0}(z, x)\right| \leq \widehat{a}(z)+\widehat{C}|x|^{r-1} \text { for a.e. } z \in \Omega, \text { all } x \in \mathbb{R}
$$

with $\widehat{a} \in L^{\infty}(\Omega)_{+}, \widehat{C}>0$ and $1<r<p^{*}$, where

$$
p^{*}= \begin{cases}\frac{N p}{N-p} & \text { if } p<N \\ +\infty & \text { if } N \leq p .\end{cases}
$$

Let $F_{0}(z, x)=\int_{0}^{x} f_{0}(z, s) d s$ and consider the $C^{1}$-functional $\varphi_{0}: W_{0}^{1, p}(\Omega) \rightarrow$ $\mathbb{R}$ defined by

$$
\varphi_{0}(u)=\int_{\Omega} G(z, D u) d z-\int_{\Omega} F_{0}(z, u) d z \quad \text { for all } u \in W_{0}^{1, p}(\Omega) .
$$

The next result was first proved by Brezis and Nirenberg [6] for $G(z, y)=$ $G(y)=\frac{1}{2}\|y\|^{2}$ (the potential for the Laplace differential operator). It was extended by Garcia Azorero et al. [15] to the case where $G(z, y)=G(y)=$ 
$\frac{1}{p}\|y\|^{p}$ with $1<p<\infty$ (the potential for the $p$-Laplace differential operator); see also Guo and Zhang [17] where $p \geq 2$. Our result is a generalization and also provides an alternative simpler proof.

Proposition 2. If $u_{0} \in W_{0}^{1, p}(\Omega)$ is a local $C_{0}^{1}(\bar{\Omega})$-minimizer of $\varphi_{0}$, i.e., there exists $\rho_{0}>0$ such that

$$
\varphi_{0}\left(u_{0}\right) \leq \varphi_{0}\left(u_{0}+h\right) \quad \text { for all } h \in C_{0}^{1}(\bar{\Omega}),\|h\|_{C_{0}^{1}(\bar{Z})} \leq \rho_{0},
$$

then $u_{0} \in C_{0}^{1}(\bar{\Omega})$ and it is also a local $W_{0}^{1, p}(\Omega)$-minimizer of $\varphi_{0}$, i.e., there exists $\rho_{1}>0$ such that

$$
\varphi_{0}\left(u_{0}\right) \leq \varphi_{0}\left(u_{0}+h\right) \quad \text { for all } h \in W_{0}^{1, p}(\Omega) \text { with }\|h\| \leq \rho_{1} .
$$

Proof. Let $h \in C_{0}^{1}(\bar{\Omega})$ and $t>0$ small. Then by hypothesis $\varphi_{0}\left(u_{0}\right) \leq \varphi_{0}\left(u_{0}+\right.$ th) hence

$$
\left\langle\varphi_{0}^{\prime}\left(u_{0}\right), h\right\rangle \geq 0
$$

Since $h \in C_{0}^{1}(\bar{\Omega})$ is arbitrary and $C_{0}^{1}(\bar{\Omega})$ is dense in $W_{0}^{1, p}(\Omega)$, from (2.3) we infer that $\varphi_{0}^{\prime}\left(u_{0}\right)=0$, hence

$$
V\left(u_{0}\right)=N_{f_{0}}\left(u_{0}\right) \text { where } N_{f_{0}}(u)(.)=f_{0}(., u(.)) \quad \text { for all } u \in W_{0}^{1, p}(\Omega) \text {, }
$$

therefore

$$
-\operatorname{div} a\left(z, D u_{0}(z)\right)=f_{0}\left(z, u_{0}(z)\right) \text { a.e. in } \Omega,\left.u_{0}\right|_{\partial \Omega}=0 .
$$

From Theorem 7.1, p. 286 of Ladyzhenskaya and Uraltseva [24] and (2.4) it follows that $u_{0} \in L^{\infty}(\Omega)$. Then we apply Theorem 1 of Lieberman [26] to conclude that $u_{0} \in C_{0}^{1, \beta}(\bar{\Omega})(0<\beta<1)$.

To show that $u_{0}$ is also a local $W_{0}^{1, p}(\Omega)$ minimizer of $\varphi_{0}$, we argue indirectly. So, suppose that $u_{0}$ is not a local $W_{0}^{1, p}(\Omega)$ minimizer of $\varphi_{0}$. Let $\varepsilon>0$ and

$$
\bar{B}_{\varepsilon}^{r}=\left\{u \in W_{0}^{1, p}(\Omega):\|u\|_{r} \leq \varepsilon\right\}
$$

where $\|\cdot\|_{r}$ denotes the norm of the Lebesgue space $L^{r}(\Omega)$. We consider the following minimization problem

$$
\inf \left\{\varphi_{0}\left(u_{0}+h\right): h \in \bar{B}_{\varepsilon}^{r}\right\}=m_{0}^{\varepsilon}>-\infty .
$$

Since by hypothesis $u_{0}$ is not a local $W_{0}^{1, p}(\Omega)$ minimizer of $\varphi_{0}$, we have

$$
m_{0}^{\varepsilon}<\varphi_{0}\left(u_{0}\right) \text { for } \varepsilon>0 \text { small. }
$$

Let $\left\{h_{n}\right\}_{n \geq 1} \subseteq \bar{B}_{\varepsilon}^{r}$ be a minimizing sequence for the problem (2.5). From Corollary 1 it follows that $\left\{h_{n}\right\}_{n \geq 1} \subseteq W_{0}^{1, p}(\Omega)$ is bounded, So, we may assume that

$$
h_{n} \stackrel{w}{\rightarrow} h_{\varepsilon} \text { in } W_{0}^{1, p}(\Omega) \text { and } h_{n} \rightarrow h_{\varepsilon} \text { in } L^{r}(\Omega) .
$$

Clearly $\varphi_{0}$ is sequentially weakly lower semicontinuous. Hence by virtue of (2.7) we have

$$
\varphi_{0}\left(u_{0}+h_{\varepsilon}\right) \leq \liminf _{n \rightarrow \infty} \varphi_{0}\left(u_{0}+h_{n}\right)=m_{0}^{\varepsilon} \text { and } h_{\varepsilon} \in \bar{B}_{\varepsilon}^{r},
$$


therefore

$$
\varphi_{0}\left(u_{0}+h_{\varepsilon}\right)=m_{0}^{\varepsilon} .
$$

So, in problem (2.5) the infimum is attained at some $h_{\varepsilon} \in \bar{B}_{\varepsilon}^{r} \backslash\{0\}$. Invoking the Lagrange multiplier rule (see, for example, Ioffe and Tichomirov [19, p. 74], we can find $\lambda_{\varepsilon} \leq 0$ such that

$$
\varphi_{0}^{\prime}\left(u_{0}+h_{\varepsilon}\right)=V_{0}\left(u_{0}+h_{\varepsilon}\right)-N_{f_{0}}\left(u_{0}+h_{\varepsilon}\right)=\lambda_{\varepsilon}\left|h_{\varepsilon}\right|^{r-2} h_{\varepsilon},
$$

hence

$$
\begin{aligned}
&-\operatorname{div} a\left(z, D\left(u_{0}+h_{\varepsilon}\right)(z)\right)= f_{0}\left(z,\left(u_{0}+h_{\varepsilon}\right)(z)\right)+\lambda_{\varepsilon}\left|h_{\varepsilon}(z)\right|^{r-2} h_{\varepsilon}(z) \\
& \text { a.e. in } \Omega,\left.h_{\varepsilon}\right|_{\partial \Omega}=0 .
\end{aligned}
$$

From (2.4) and (2.8) it follows that

$$
\begin{aligned}
-\operatorname{div}\left[a\left(z, D\left(u_{0}+h_{\varepsilon}\right)(z)\right)-a\left(z, D u_{0}(z)\right)\right]= & f_{0}\left(z,\left(u_{0}+h_{\varepsilon}\right)(z)\right)-f_{0}\left(z, u_{0}(z)\right) \\
& +\lambda_{\varepsilon}\left|h_{\varepsilon}(z)\right|^{r-2} h_{\varepsilon}(z) \text { a.e. in } \Omega .
\end{aligned}
$$

Case 1. Suppose $\lambda_{\varepsilon} \in[-1,0]$ for all $\varepsilon \in(0,1]$.

We set

$$
v_{\varepsilon}(z)=\left(u_{0}+h_{\varepsilon}\right)(z) \text { and } \xi_{\varepsilon}(z, y)=a(z, y)+a\left(z, D u_{0}(z)\right) .
$$

Then (2.9) becomes

$$
\begin{aligned}
-\operatorname{div} \xi_{\varepsilon}\left(z, D v_{\varepsilon}(z)\right)= & f_{0}\left(z, v_{\varepsilon}(z)\right)-f_{0}\left(z, u_{0}(z)\right) \\
& +\lambda_{\varepsilon}\left|\left(v_{\varepsilon}-u_{0}\right)(z)\right|^{r-2}\left(v_{\varepsilon}-u_{0}\right)(z) \text { a.e. in } \Omega .
\end{aligned}
$$

Recall that $u_{0} \in C_{0}^{1, \beta}(\bar{\Omega})$. From (2.10) and [24] (Theorem 7.1, p. 286), we have $v_{\varepsilon} \in L^{\infty}(\Omega)$ and $\left\|v_{\varepsilon}\right\|_{\infty} \leq M_{1}$ for some $M_{1}>0$, all $\varepsilon \in(0,1]$. Invoking Theorem 1 of Lieberman [26], we can find $\gamma \in(0,1]$ and $M_{2}>0$ such that

$$
v_{\varepsilon} \in C_{0}^{1, \gamma}(\bar{\Omega}) \text { and }\left\|v_{\varepsilon}\right\|_{C_{0}^{1, \gamma}(\bar{\Omega})} \leq M_{2} \quad \text { for all } \varepsilon \in(0,1] .
$$

Case 2. Suppose $\lambda_{\varepsilon_{n}}<-1$ for all $n \geq 1$ with $\varepsilon_{n} \rightarrow 0+$.

In this case we set

$$
\widehat{\xi}_{\varepsilon_{n}}(z, y)=\frac{1}{\left|\lambda_{\varepsilon_{n}}\right|}\left[a\left(z, D u_{0}(z)+y\right)-a\left(z, D u_{0}(z)\right)\right] .
$$

Then (2.9) becomes

$$
\begin{aligned}
-\operatorname{div} \widehat{\xi}_{\varepsilon_{n}}\left(z, D h_{\varepsilon_{n}}(z)\right)= & \frac{1}{\left|\lambda_{\varepsilon_{n}}\right|}\left[f_{0}\left(z,\left(u_{0}+h_{\varepsilon_{n}}\right)(z)\right)-f_{0}\left(z, u_{0}(z)\right)\right] \\
& -\left|h_{\varepsilon_{n}}(z)\right|^{r-2} h_{\varepsilon_{n}}(z) \text { a.e. in } \Omega .
\end{aligned}
$$

As before, from [24] and [26] (Theorem 1), we can find $\widehat{\gamma} \in(0,1]$ and $M_{3}>0$ such that

$$
h_{\varepsilon_{n}} \in C_{0}^{1, \widehat{\gamma}}(\bar{\Omega}) \text { and }\left\|h_{\varepsilon_{n}}\right\|_{C_{0}^{1, \hat{\gamma}}(\bar{\Omega})} \leq M_{3} \quad \text { for all } n \geq 1 .
$$


From (2.11) (2.12) and the compact embedding of $C_{0}^{1, \gamma^{\prime}}(\bar{\Omega})\left(0<\gamma^{\prime} \leq 1\right)$ into $C_{0}^{1}(\bar{\Omega})$, we see that we can find a sequence $\left\{h_{\varepsilon_{n}}\right\}_{n \geq 1}$ such that

$$
u_{0}+h_{\varepsilon_{n}} \rightarrow u_{0} \text { in } C_{0}^{1}(\bar{\Omega}) .
$$

Since $u_{0}$ is a local $C_{0}^{1}(\bar{\Omega})$-minimizer of $\varphi_{0}$, we have

$$
\varphi_{0}\left(u_{0}\right) \leq \varphi_{0}\left(u_{0}+h_{\varepsilon_{n}}\right) \quad \text { for all } n \geq n_{0}(\text { see }(2.13)) .
$$

On the other hand, from the choice of $\left\{h_{\varepsilon_{n}}\right\}_{n \geq 1}$ we have

$$
m_{0}^{\varepsilon_{n}}=\varphi_{0}\left(u_{0}+h_{\varepsilon_{n}}\right)<\varphi_{0}\left(u_{0}\right) \quad \text { for all } n \geq 1 \text {. }
$$

Comparing (2.14) and (2.15) we reach a contradiction.

In what follows, by $\widehat{\lambda}_{1}$ we denote the first eigenvalue of the negative Dirichlet $p$-Laplacian $\left(-\triangle_{p}, W_{0}^{1, p}(\Omega)\right)$. It admits a variational characterization:

$$
\widehat{\lambda}_{1}=\inf \left\{\frac{\|D u\|_{p}^{p}}{\|u\|_{p}^{p}}: u \in W_{0}^{1, p}(\Omega), u \neq 0\right\} .
$$

The infimum in (2.16) is attained in the eigenspace of $\widehat{\lambda}_{1}$, which is a simple and isolated eigenvalue. By $\widehat{u}_{1}$ we denote the $L^{p}$-normalized positive eigenfunction corresponding to $\hat{\lambda}_{1}$ (see $\left.(2.16)\right)$. The nonlinear regularity theory (see $[24,26]$ ) implies that $\widehat{u}_{1} \in \mathcal{C}_{+} \backslash\{0\}$. In fact, by the nonlinear strong maximum principle of Vazquez [34], $\widehat{u}_{1} \in \operatorname{int} \mathcal{C}_{+}$.

Also, if $\sigma(p)$ denotes the spectrum of $\left(-\triangle_{p}, W_{0}^{1, p}(\Omega)\right)$ (i.e., the set of eigenvalues of the negative Dirichlet $p$-Laplacian), then since $\widehat{\lambda}_{1}$ is isolated and $\sigma(p)$ is closed, we can define

$$
\widehat{\lambda}_{2}=\inf \left\{\widehat{\lambda}: \widehat{\lambda} \in \sigma(p) \backslash\left\{\widehat{\lambda}_{1}\right\}\right\} .
$$

This is the second eigenvalue of $\left(-\triangle_{p}, W_{0}^{1, p}(\Omega)\right)$ and admits the following minimax characterization

$$
\widehat{\lambda}_{2}=\inf _{\gamma_{0} \in \Gamma_{0}} \max _{-1 \leq t \leq 1}\left\|D \gamma_{0}(t)\right\|_{p}^{p},
$$

where

$$
\Gamma_{0}=\left\{\gamma_{0} \in C([-1,1], M): \gamma_{0}(-1)=-\widehat{u}_{1}, \gamma_{0}(1)=\widehat{u}_{1}\right\},
$$

with

$$
M=W_{0}^{1, p}(\Omega) \cap \partial B_{1}^{L^{p}}, \quad \partial B_{1}^{L^{p}}=\left\{u \in L^{p}(\Omega):\|u\|_{p}=1\right\}
$$

(see Cuesta et al. [8]).

As we already indicated, for every $u \in W_{0}^{1, p}(\Omega),\|u\|=\|D u\|_{p}$. Also, for all $r \in \mathbb{R}$, we set

$$
r^{ \pm}:=\max \{ \pm r, 0\} .
$$

Finally by $|\cdot|_{N}$ we denote the Lebesgue measure on $\mathbb{R}^{N}$. 


\section{Solutions of constant sign}

The hypotheses on the reaction $f(z, x)$ are the following:

$\mathbf{H}_{\mathbf{1}}(\mathbf{f}): f: \Omega \times \mathbb{R} \rightarrow \mathbb{R}$ is a Carathéodory function such that $f(z, 0)=0$ for a.e. $z \in \Omega$ and:

(i) $|f(z, x)| \leq a(z)+C|x|^{r-1}$ for a.e. $z \in \Omega$, all $x \in \mathbb{R}$, with $a \in$ $L^{\infty}(\Omega)_{+}, C>0$ and $p<r<p^{*}$;

(ii) if $F(z, x)=\int_{0}^{x} f(z, s) d s$ and $\sigma(z, x)=f(z, x) x-p F(z, x)$, then

$$
\lim _{x \rightarrow \pm \infty} \frac{F(z, x)}{|x|^{p}}=+\infty \text { uniformly for a.e. } z \in \Omega
$$

and there exists $\mu \geq 1, \mu \in\left(q, p^{*}\right)$ with $q=(r-p) \max \left\{\frac{N}{p}, 1\right\}$ and $\eta_{0}>0$ such that

$$
\lim _{x \rightarrow \pm \infty} \frac{\sigma(z, x)}{|x|^{\mu}} \geq \eta_{0}>0 \text { uniformly for a.e. } z \in \Omega
$$

(iii) there exists $\theta \in L^{\infty}(\Omega)_{+}, \theta(z) \geq \frac{C_{1}}{p-1} \widehat{\lambda}_{1}$ a.e. in $\Omega, \theta \neq \frac{C_{1}}{p-1} \widehat{\lambda}_{1}$ and

$$
\liminf _{x \rightarrow 0} \frac{p F(z, x)}{|x|^{p}} \geq \theta(z) \text { uniformly for a.e. } z \in \Omega \text {; }
$$

(iv) there exist $C_{-}<0<C_{+}$such that

$$
\text { ess } \sup f\left(., C_{+}\right) \leq 0 \leq \underset{\Omega}{\operatorname{essinf}} f\left(., C_{-}\right) ;
$$

(v) for every $\rho>0$, there exists $\xi_{\rho}>0$ such that for a.e. $z \in \Omega$

$$
x \rightarrow f(z, x)+\xi_{\rho}|x|^{p-2} x
$$

is nondecreasing on $[-\rho, \rho]$.

Remarks. Hypothesis $\mathbf{H}_{1}(f)(i i)$ implies that the primitive function $F(z,$.$) is$ $p$-superlinear near $\pm \infty$. Similar conditions were used by Costa and Magalhaes [7] (Dirichlet equations) and Fei [14] (Hamiltonian systems). Evidently, if

$$
\lim _{x \rightarrow \pm \infty} \frac{f(z, x)}{|x|^{p-2} x}=+\infty \quad \text { uniformly for a.e. } z \in \Omega,
$$

then

$$
\lim _{x \rightarrow \pm \infty} \frac{F(z, x)}{|x|^{p}}=+\infty \quad \text { uniformly for a.e. } z \in \Omega .
$$

So, our problem is $(p-1)$-superlinear, but instead of the AR-condition we use hypothesis $\mathbf{H}_{1}(f)(i i)$. This incorporates in our analytical framework "superlinear" forcing terms with slower growth near $\pm \infty$, which are otherwise excluded by the AR condition (see the Examples that follow).

Hypothesis $\mathbf{H}_{1}(f)(i v)$ implies that $f(z,$.$) has zeros. As we explain later,$ in the case of a $z$-independent map (i.e., $a(z, y)=a(y))$ this hypothesis can have a more general form by replacing $C_{ \pm}$by certain constant sign $C^{1}(\bar{\Omega})$ functions. Finally note that no sign condition is imposed on $f(z,$.$) .$ 
Example. The following functions satisfy hypotheses $\mathbf{H}_{1}(f)$. (For the sake of simplicity, we drop the $z$-dependence.)

$f_{1}(x)=\theta|x|^{p-2} x+|x|^{r-2} x-(\theta+1)|x|^{\tau-2} x \quad$ with $\theta>\frac{C_{1}}{p-1} \widehat{\lambda}_{1}, 1<p<\tau<r<p^{*}$. $f_{2}(x)=\left\{\begin{array}{ll}\theta|x|^{p-2} x-|x|^{r-2} x & \text { if }|x| \leq 1 \\ |x|^{p-2} x\left(\ln |x|+\frac{1}{p}\right)-\frac{1}{p}|x|^{q-2} x & \text { if }|x|>1\end{array}\right.$ with $1<q<p<r<\infty$ and $\theta$ as in $f_{1}$.

Note that $f_{1}$ satisfies the AR-condition, but $f_{2}$ does not.

Let $\varphi: W_{0}^{1, p}(\Omega) \rightarrow \mathbb{R}$ be the energy functional for the problem (1) defined by

$$
\varphi(u)=\int_{\Omega} G(z, D u) d z-\int_{\Omega} F(z, u) d z \quad \text { for all } u \in W_{0}^{1, p}(\Omega) .
$$

Evidently $\varphi \in C^{1}\left(W_{0}^{1, p}(\Omega)\right)$.

Proposition 3. If hypotheses $\mathbf{H}(\mathbf{a})(i)$-(iii) and $\mathbf{H}_{1}(f)(i),(i i i),(i v),(v)$ hold, then problem (1.1) has at least two constant sign smooth solutions $u_{0} \in$ int $\mathcal{C}_{+}$ and $v_{0} \in-$ int $\mathcal{C}_{+}$such that

$$
C_{-}<v_{0}(z) \leq 0 \leq u_{0}(z)<C_{+} \quad \text { for all } z \in \bar{\Omega}
$$

and both are local minimizers of $\varphi$.

Proof. First we produce the positive solution. To this end, we introduce the following truncation of $f(z,$.$) :$

$$
\widehat{f}_{+}(z, x)= \begin{cases}0 & \text { if } x<0 \\ f(z, x) & \text { if } 0 \leq x \leq C_{+} \\ f\left(z, C_{+}\right) & \text {if } C_{+}<x\end{cases}
$$

This is a Carathéodory function. We set

$$
\widehat{F}_{+}(z, x)=\int_{0}^{x} \widehat{f}_{+}(z, s) d s
$$

and consider the $C^{1}$-functional $\widehat{\varphi}_{+}: W_{0}^{1, p}(\Omega) \rightarrow \mathbb{R}$ defined by

$$
\widehat{\varphi}_{+}(u)=\int_{\Omega} G(z, D u) d z-\int_{\Omega} \widehat{F}_{+}(z, u) d z \quad \text { for all } u \in W_{0}^{1, p}(\Omega) .
$$

Corollary 1 and (3.1) imply that $\widehat{\varphi}_{+}$is coercive. Moreover, by using the Sobolev embedding theorem, we can check that $\widehat{\varphi}_{+}$is sequentially weakly lower semicontinuous. So, by the Weierstrass theorem, we can find $u_{0} \in W_{0}^{1, p}(\Omega)$ such that

$$
\widehat{\varphi}_{+}\left(u_{0}\right)=\inf \left\{\widehat{\varphi}_{+}(u): u \in W_{0}^{1, p}(\Omega)\right\}=\widehat{m}_{+} .
$$

By virtue of hypothesis $\mathbf{H}_{1}(f)($ iii $)$, given $\varepsilon>0$ we can find $\delta=\delta(\varepsilon) \in\left(0, C_{+}\right)$ such that

$$
F(z, x) \geq \frac{1}{p}(\theta(z)-\varepsilon)|x|^{p} \text { for a.e. } z \in \Omega \text {, all }|x| \leq \delta .
$$


Let $\widehat{u}_{1} \in$ int $\mathcal{C}_{+}$be the $L^{p}$-normalized principal eigenfunction of $\left(-\triangle_{p}, W_{0}^{1, p}(\Omega)\right)$ (see Sect. 2) and let $t \in(0,1)$ be small, such that $t \widehat{u}_{1}(z) \in[0, \delta]$ for all $z \in \bar{\Omega}$. Then (see Corollary 1 and 3.3 )

$$
\begin{aligned}
\widehat{\varphi}_{+}\left(t \widehat{u}_{1}\right) & =\int_{\Omega} G\left(z, t D \widehat{u}_{1}\right) d z-\int_{\Omega} \widehat{F}_{+}\left(z, t \widehat{u}_{1}\right) d z \\
& \leq \frac{C_{1} t^{p}}{p(p-1)}\left\|D \widehat{u}_{1}\right\|_{p}^{p}-\frac{t^{p}}{p} \int_{\Omega}(\theta(z)-\varepsilon) \widehat{u}_{1}^{p} d z \\
& =\frac{t^{p}}{p} \int_{\Omega}\left(\frac{C_{1} \widehat{\lambda}_{1}}{p-1}-\theta(z)\right) \widehat{u}_{1}^{p} d z+\frac{\varepsilon t^{p}}{p}\left(\text { since }\left\|\widehat{u}_{1}\right\|_{p}^{p}=1\right) .
\end{aligned}
$$

Note that

$$
\int_{\Omega}\left(\frac{C_{1} \widehat{\lambda}_{1}}{p-1}-\theta(z)\right) \widehat{u}_{1}^{p} d z<0
$$

(see $\mathbf{H}_{1}(f)($ iii $)$ and recall that $\widehat{u}_{1} \in$ int $\left.\mathcal{C}_{+}\right)$. So, for $\varepsilon>0$ small, from (3.4) it follows

$$
\widehat{\varphi}_{+}\left(t \widehat{u}_{1}\right)<0,
$$

hence

$$
\widehat{\varphi}_{+}\left(u_{0}\right)=\widehat{m}_{+}<0=\widehat{\varphi}_{+}(0)
$$

therefore

$$
u_{0} \neq 0 \text {. }
$$

From (3.2) we have

$$
\widehat{\varphi}_{+}^{\prime}\left(u_{0}\right)=0
$$

hence

$$
V\left(u_{0}\right)=N_{\widehat{f}_{+}}\left(u_{0}\right) \text { where } N_{\widehat{f}_{+}}(u)(.)=\widehat{f}_{+}(., u(.)) \quad \text { for all } u \in W_{0}^{1, p}(\Omega) .
$$

On $(3.5)$ we first act with $-u_{0}^{-} \in W_{0}^{1, p}(\Omega)$. Then

$$
\int_{\Omega}\left(a\left(z, D u_{0}\right),-D u_{0}^{-}\right)_{\mathbb{R}^{N}} d z=0(\text { see }(3.1)) \text {. }
$$

Recall that

$$
D u_{0}^{-}(z)=\left\{\begin{array}{ll}
-D u_{0}(z) & \text { if } u_{0}(z)<0 \\
0 & \text { if } u_{0}(z) \geq 0
\end{array} .\right.
$$

Then, from (3.6) and Lemma $1(c)$, we have

$$
\frac{C_{0}}{p-1}\left\|D u_{0}^{-}(z)\right\|_{p}^{p} \leq 0
$$

hence

$$
u_{0} \geq 0, u_{0} \neq 0
$$


Next on (3.5) we act with $\left(u_{0}-C_{+}\right)^{+} \in W_{0}^{1, p}(\Omega)$. We obtain

$$
\begin{aligned}
\int_{\Omega}\left(a\left(z, D u_{0}\right), D\left(u_{0}-C_{+}\right)^{+}\right)_{\mathbb{R}^{N}} d z & =\int_{\Omega} \widehat{f}_{+}\left(z, u_{0}\right)\left(u_{0}-C_{+}\right)_{\mathbb{R}^{N}}^{+} d z \\
& =\int_{\Omega} f\left(z, C_{+}\right)\left(u_{0}-C_{+}\right)_{\mathbb{R}^{N}}^{+} d z(\operatorname{see}(3.1)) \\
& \leq 0\left(\operatorname{see} \mathbf{H}_{1}(f)(i v)\right),
\end{aligned}
$$

hence

$$
\int_{\left\{u_{0}>C_{+}\right\}}\left(a\left(z, D u_{0}\right)-a\left(z, D C_{+}\right), D u_{0}-D C_{+}\right)_{\mathbb{R}^{N}} d z \leq 0,
$$

which yields

$$
\left|\left\{u_{0}>C_{+}\right\}\right|_{N}=0(\text { see Lemma } 1(a))
$$

and therefore

$$
u_{0} \leq C_{+} \text {a.e. in } \Omega \text {. }
$$

So, we have

$$
u_{0} \in\left[0, C_{+}\right]:=\left\{u \in W_{0}^{1, p}(\Omega): 0 \leq u(z) \leq C_{+} \text {a.e. in } \Omega\right\} .
$$

Hence, (3.5) becomes

$$
V\left(u_{0}\right)=N_{f}\left(u_{0}\right) \text { with } N_{f}(u)(.)=f(., u(.)) \quad \text { for all } u \in W_{0}^{1, p}(\Omega)
$$

(see $(3.1))$, therefore

$$
-\operatorname{div} a\left(z, D u_{0}(z)\right)=f_{0}\left(z, u_{0}(z)\right) \text { a.e. in } \Omega,\left.u_{0}\right|_{\partial \Omega}=0 \text {. }
$$

The nonlinear regularity theory (see Lieberman [26]) implies that $u_{0} \in$ $\mathcal{C}_{+} \backslash\{0\}$.

Let $\rho=C_{+}>0$ and let $\xi_{\rho}$ as postulated by $\mathbf{H}_{1}(f)(v)$. Then $-\operatorname{div} a\left(z, D u_{0}(z)\right)+\xi_{\rho} u_{0}(z)^{p-1}=f_{0}\left(z, u_{0}(z)\right) \xi_{\rho} u_{0}(z)^{p-1} \geq 0 \quad$ a.e. in $\Omega$, hence

$$
\operatorname{div} a\left(z, D u_{0}(z)\right) \leq \xi_{\rho} u_{0}(z)^{p-1} \text { a.e. in } \Omega \text {. }
$$

Invoking Theorem 1.2 of Zhang [35], we infer that $u_{0} \in$ int $\mathcal{C}_{+}$. Also since $u_{0}(z) \leq C_{+}$for all $z \in \bar{\Omega}$, using hypothesis $\mathbf{H}_{1}(f)(v)$, we have

$$
-\operatorname{div} a\left(z, D u_{0}(z)\right)-\xi_{\rho}\left(C_{+}^{p-1}-u_{0}(z)^{p-1}\right) \leq 0 \text { for } \quad \text { a.e. } z \in \Omega,
$$

hence

$$
\operatorname{div} a\left(z, D\left(C_{+}-u_{0}(z)\right)\right)-\xi_{\rho}\left(C_{+}^{p-1}-u_{0}(z)^{p-1}\right) \leq 0 \text { for a.e. } z \in \Omega
$$

(recall that $a(z,$.$) is odd, see \mathbf{H}(a))$.

If $1<p \leq 2$, then

$$
C_{+}^{p-1}-u_{0}(z)^{p-1} \leq \alpha_{1}\left(C_{+}-u_{0}(z)\right)^{p-1} \text { for some } \alpha_{1}>0
$$


(see [15], p. 391). Then

$$
\operatorname{div} a\left(z, D\left(C_{+}-u_{0}(z)\right)\right)-\xi_{\rho} \alpha_{1}\left(C_{+}-u_{0}(z)\right)^{p-1} \leq 0 \text { for a.e. } z \in \Omega \text {. }
$$

Invoking Theorem 2.5.1, p. 34, of Pucci and Serrin [30] (see also Damascelli [10], p. 495) from (3.8) we infer that

$$
u_{0}(z)<C_{+} \quad \text { for all } z \in \bar{\Omega} .
$$

Now suppose $2<p<\infty$. Then

$$
C_{+}^{p-1}-u_{0}(z)^{p-1} \leq \alpha_{2}\left(C_{+}-u_{0}(z)\right) \text { for some } \alpha_{2}>0
$$

(see [15], p. 391). So, from (3.7) we have

$$
0 \leq-\operatorname{div} a\left(z, D\left(C_{+}-u_{0}(z)\right)\right)+\alpha_{2}\left(C_{+}-u_{0}(z)\right) \text { for a.e. } z \in \Omega .
$$

Let

$$
K=\left\{z \in \Omega: u_{0}(z)=C_{+}\right\} .
$$

Evidently $K$ is a compact subset of $\Omega$ (recall that $\left.u_{0}\right|_{\partial \Omega}=0$ ). Also, if $z \in K$, then from (3.9) and the Harnack inequality of Pucci and Serrin [30], p. 154), we know that there exists $\rho>0$ such that

$$
B_{\rho}(z)=\left\{z^{\prime} \in \Omega:\left\|z-z^{\prime}\right\|<\rho\right\} \subseteq K .
$$

So, $K$ is open in $\Omega$. Therefore

$$
K=\Omega \text { or } K=\varnothing .
$$

Since $K \neq \Omega$, we must have $K=\varnothing$, and so again we conclude that

$$
u_{0}(z)<C_{+} \quad \text { for all } z \in \bar{\Omega} .
$$

Therefore, in both cases we see that $u_{0} \in$ int $_{C_{0}^{1}(\bar{\Omega})}\left[0, C_{+}\right]$. Note that $\left.\varphi\right|_{\left[0, C_{+}\right]}=$ $\left.\widehat{\varphi}_{+}\right|_{\left[0, C_{+}\right]}($see $(3.1))$. It follows that $u_{0}$ is a local $C_{0}^{1}(\bar{\Omega})$-minimizer of $\varphi$ and so, invoking Proposition 2 , we conclude that $u_{0}$ is a local $W_{0}^{1, p}(\Omega)$-minimizer of $\varphi$.

Similarly, by introducing the truncation of $f(z,$.$) at \left\{0, C_{-}\right\}$and reasoning as above, we produce a second constant sign solution $v_{0} \in-$ int $\mathcal{C}_{+}$, such that $v_{0}(z)>C_{-}$for all $z \in \bar{\Omega}$ and $v_{0}$ is a local $W_{0}^{1, p}(\Omega)$-minimizer of $\varphi$.

Remarks. If $a$ is $z$-independent (i.e., $a(z, y)=a(y))$ then Proposition 3 remains valid if hypothesis $\mathbf{H}_{1}(f)(i v)$ is replaced by the following more general one:

$\mathbf{H}_{1}(f)(i v)^{\prime}$ : there exist functions $w_{ \pm} \in C^{1}(\bar{\Omega})$ such that

$$
\begin{aligned}
& w_{-}(z)<0<w_{+}(z) \quad \text { for all } z \in \bar{\Omega} \\
& \text { ess } \sup f\left(., w_{+}(.)\right) \leq 0 \leq \text { ess inff }\left(., w_{+}(.)\right) \text {and } \\
& \Omega \\
& V\left(w_{-}\right) \leq 0 \leq V\left(w_{+}\right) \text {in the sense of distributions. }
\end{aligned}
$$


In this case, to show that $u_{0}(z)<w_{+}(z)$ and $w_{-}(z)<v_{0}(z)$ for all $z \in \bar{\Omega}$, we argue as in the strong comparison principle of Arcoya and Ruiz [5] (cf. Proposition 2.6 and its proof; see also Tolksdorf [33], Proposition 3.32).

Using these two solutions and variational methods coupled with truncation techniques, we can produce two additional constant sign smooth solutions.

Proposition 4. If hypotheses $\mathbf{H}(\mathbf{a})$ and $\mathbf{H}_{1}(f)$ hold, then problem (1.1) has two additional constant sign smooth solutions $\widehat{u} \in$ int $\mathcal{C}_{+}$and $\widehat{v} \in-$ int $\mathcal{C}_{+}$, such that

$$
u_{0}(z) \leq \widehat{u}(z) \quad \text { for all } z \in \bar{\Omega}, u_{0} \neq \widehat{u}
$$

and

$$
\widehat{v}(z) \leq v_{0}(z) \quad \text { for all } z \in \bar{\Omega}, v_{0} \neq \widehat{v} .
$$

Proof. First we produce the second positive smooth solution. To this end, let $u_{0} \in$ int $\mathcal{C}_{+}$be the positive smooth solution from Proposition 6 and consider the following truncation of $f(z,$.$) :$

$$
h_{+}(z, x)= \begin{cases}f\left(z, u_{0}(z)\right) & \text { if } x \leq u_{0}(z) \\ f(z, x) & \text { if } u_{0}(z)<x .\end{cases}
$$

This is a Carathéodory function. We set

$$
H_{+}(z, x)=\int_{0}^{x} h_{+}(z, s) d s
$$

and consider the $C^{1}$-functional $\psi_{+}: W_{0}^{1, p}(\Omega) \rightarrow \mathbb{R}$ defined by

$$
\psi_{+}(u)=\int_{\Omega} G(z, D u) d z-\int_{\Omega} H_{+}(z, u) d z \quad \text { for all } u \in W_{0}^{1, p}(\Omega) .
$$

Claim 1. $\psi_{+}$satisfies the $C$-condition. Let $\left\{u_{n}\right\}_{n \geq 1} \subseteq W_{0}^{1, p}(\Omega)$ be a sequence such that

$$
\left|\psi_{+}\left(u_{n}\right)\right| \leq M_{1} \quad \text { for some } M_{1}>0, \text { all } n \geq 1
$$

and

$$
\left(1+\left\|u_{n}\right\|\right) \psi_{+}^{\prime}\left(u_{n}\right) \rightarrow 0 \quad \text { in } W^{-1, p^{\prime}}(\Omega) \quad \text { as } n \rightarrow \infty .
$$

From (3.12) we have

$$
\left|\left\langle\psi_{+}^{\prime}\left(u_{n}\right), v\right\rangle\right| \leq \frac{\varepsilon_{n}\|v\|}{1+\left\|u_{n}\right\|} \quad \text { for all } v \in W_{0}^{1, p}(\Omega), \text { with } \varepsilon_{n} \rightarrow 0^{+},
$$

hence

$$
\left|\left\langle V\left(u_{n}\right), v\right\rangle-\int_{\Omega} h_{+}\left(z, u_{n}\right) v d z\right| \leq \frac{\varepsilon_{n}\|v\|}{1+\left\|u_{n}\right\|} \quad \text { for all } n \geq 1 .
$$

In (3.13) first we choose $v=-u_{n}^{-} \in W_{0}^{1, p}(\Omega)$. Then from Lemma $1(c)$ and (3.10) we have

$$
\frac{C_{0}}{p-1}\left\|D u_{n}^{-}\right\|_{p}^{p} \leq \varepsilon_{n} \quad \text { for all } n \geq 1
$$


hence

$$
u_{n}^{-} \rightarrow 0 \text { in } W_{0}^{1, p}(\Omega) .
$$

Then from (3.11), (3.14) and Corollary 1, we have

$$
\int_{\Omega} p G\left(z, D u_{n}^{+}\right) d z-\int_{\Omega} p H_{+}\left(z, u_{n}^{+}\right) d z \leq M_{2} \text { for some } M_{2}>0 \text {, all } n \geq 1 \text {. }
$$

Moreover, if in (3.13) we choose $v=u_{n}^{+} \in W_{0}^{1, p}(\Omega)$ then

$$
-\int_{\Omega}\left(a\left(z, D u_{n}^{+}\right), D u_{n}^{+}\right)_{\mathbb{R}^{N}} d z+\int_{\Omega} h_{+}\left(z, u_{n}^{+}\right) u_{n}^{+} d z \leq \varepsilon_{n} \quad \text { for all } n \geq 1 .
$$

Adding (3.15) and (3.16), we obtain

$$
\begin{aligned}
& \int_{\Omega}\left[h_{+}\left(z, u_{n}^{+}\right) u_{n}^{+}-p H_{+}\left(z, u_{n}^{+}\right)\right] d z \\
& \quad \leq \int_{\Omega}\left[\left(a\left(z, D u_{n}^{+}\right), D u_{n}^{+}\right)_{\mathbb{R}^{N}}-p G\left(z, D u_{n}^{+}\right)\right] d z+M_{2}+\varepsilon_{n} \quad \text { for all } n \geq 1,
\end{aligned}
$$

hence

$$
\int_{\Omega}\left[h_{+}\left(z, u_{n}^{+}\right) u_{n}^{+}-p H_{+}\left(z, u_{n}^{+}\right)\right] d z \leq M_{3} \text { for some } M_{3}>0, \text { all } n \geq 1
$$

( see $\mathbf{H}(a)(v))$, therefore

$$
\int_{\Omega} \sigma\left(z, u_{n}^{+}\right) d z \leq M_{4} \quad \text { for some } M_{4}>0, \text { all } n \geq 1 \text { (see (3.10)). }
$$

Hypotheses $\mathbf{H}_{1}(f)(i),(i i)$ imply that we can find $\eta_{1} \in\left(0, \eta_{0}\right)$ and $M_{5}>0$ such that

$$
\sigma(z, x) \geq \eta_{1} x^{\mu}-M_{5} \quad \text { for a.e. } z \in \Omega, \text { all } x \geq 0 .
$$

Using this in (3.17), we see that

$$
\left\{u_{n}^{+}\right\}_{n \geq 1} \subseteq L^{\mu}(\Omega) \text { is bounded. }
$$

First assume $N \neq p$. From $\mathbf{H}_{1}(f)(i i)$ it is clear that we may assume $\mu \leq r<p^{*}$. Let $t \in[0,1)$ such that

$$
\frac{1}{r}=\frac{1-t}{\mu}+\frac{t}{p^{*}} .
$$

Then the interpolation inequality (see [16], p. 905) implies that

$$
\left\|u_{n}^{+}\right\|_{r} \leq\left\|u_{n}^{+}\right\|_{\mu}^{1-t}\left\|u_{n}^{+}\right\|_{p^{*}}^{t},
$$

hence

$$
\left\|u_{n}^{+}\right\|_{r}^{r} \leq M_{6}\left\|u_{n}^{+}\right\|^{t r} \text { for some } M_{6}>0 \text {, all } n \geq 1
$$

(see (3.18) and use the Sobolev embedding theorem). From hypothesis $\mathbf{H}_{1}(f)(i)$ we have

$$
f(z, x) x \leq a(z) x+C x^{r} \quad \text { for a.e. } z \in \Omega \text {, all } x \geq 0 .
$$


From (3.13) with $v=u_{n}^{+} \in W_{0}^{1, p}(\Omega)$, it follows that

$$
\int_{\Omega}\left(a\left(z, D u_{n}^{+}\right), D u_{n}^{+}\right)_{\mathbb{R}^{N}} d z-\int_{\Omega} h_{+}\left(z, u_{n}^{+}\right) u_{n}^{+} \leq \varepsilon_{n} \quad \text { for all } n \geq 1,
$$

hence (see Lemma $1(c),(3.20)$ and (3.10)

$$
\begin{aligned}
& \frac{C_{0}}{p-1}\left\|D u_{n}^{+}\right\|_{p}^{p} \leq M_{7}\left(1+\left\|u_{n}^{+}\right\|_{r}^{r}\right) \text { for some } M_{7}>0, \text { all } n \geq 1 \\
& \quad \leq M_{8}\left(1+\left\|u_{n}^{+}\right\|^{t r}\right) \text { for some } M_{8}>0, \text { all } n \geq 1(\text { see }(3.19))
\end{aligned}
$$

The hypothesis on $\mu$ (see $\left.\mathbf{H}_{1}(f)(i i)\right)$ implies that $t r<p$, and so, from (3.21) it follows that

$$
\left\{u_{n}^{+}\right\}_{n \geq 1} \subseteq W_{0}^{1, p}(\Omega) \text { is bounded. }
$$

If $N=p$, then by definition $p^{*}=\infty$, while from the Sobolev embedding theorem, we know that $W_{0}^{1, p}(\Omega)$ is embedded compactly in $L^{q}(\Omega)$ for all $q \in$ $[1, \infty)$. So, in the previous argument, we replace $p^{*}$ by $\widehat{p}>r$ large such that

$$
t r=\frac{\widehat{p}(r-\mu)}{\widehat{p}-\mu}<p .
$$

Then, we deduce again (3.22). From (3.14) and (3.22) it follows that $\left\{u_{n}\right\}_{n \geq 1} \subseteq$ $W_{0}^{1, p}(\Omega)$ is bounded, so, we may assume

$$
u_{n} \stackrel{w}{\rightarrow} u \text { in } W_{0}^{1, p}(\Omega) \text { and } u_{n} \rightarrow u \text { in } L^{r}(\Omega) .
$$

In (3.13) we choose $v=u_{n}-u$, pass to the limit as $n \rightarrow \infty$, and use (3.23). Then

$$
\lim _{n \rightarrow \infty}\left\langle V\left(u_{n}\right), u_{n}-u\right\rangle=0
$$

hence

$$
u_{n} \rightarrow u \text { in } W_{0}^{1, p}(\Omega)
$$

(see Proposition 1). This proves Claim 1.

Claim 2. We may assume that $u_{0} \in$ int $\mathcal{C}_{+}$is a local minimizer of $\psi_{+}$.

We consider the following truncation of $h_{+}(z,$.$) :$

$$
\widehat{h}_{+}(z, x)= \begin{cases}h_{+}(z, x) & \text { if } x \leq C_{+} \\ h_{+}\left(z, C_{+}\right) & \text {if } C_{+}<x\end{cases}
$$

(recall that $u_{0}(z)<C_{+}$for all $z \in \bar{\Omega}$ ). This is a Carathéodory function. We set

$$
\widehat{H}_{+}(z, x)=\int_{0}^{x} \widehat{h}_{+}(z, s) d s
$$

and consider the $C^{1}$-functional $\widehat{\psi}_{+}: W_{0}^{1, p}(\Omega) \rightarrow \mathbb{R}$ defined by

$$
\widehat{\psi}_{+}(u)=\int_{\Omega} G(z, D u) d z-\int_{\Omega} \widehat{H}_{+}(z, u) d z \quad \text { for all } u \in W_{0}^{1, p}(\Omega) .
$$


Evidently, $\widehat{\psi}$ is coercive (see (3.24)) and sequentially weakly lower semicontinuous. So, by the Weierstrass theorem, we can find $\widehat{u}_{0} \in W_{0}^{1, p}(\Omega)$ such that

$$
\widehat{\psi}_{+}\left(\widehat{u}_{0}\right)=\inf \left\{\widehat{\psi}_{+}(u): u \in W_{0}^{1, p}(\Omega)\right\}
$$

hence

$$
\widehat{\psi}_{+}^{\prime}\left(\widehat{u}_{0}\right)=0
$$

therefore

$$
V\left(\widehat{u}_{0}\right)=N_{\widehat{h}_{+}}\left(\widehat{u}_{0}\right) \text { where } N_{\widehat{h}_{+}}(u)(.)=\widehat{h}_{+}(., u(.)) \quad \text { for all } u \in W_{0}^{1, p}(\Omega) .
$$

On $(3.25)$ we act with $\left(u_{0}-\widehat{u}_{0}\right)^{+}$and obtain

$$
\begin{aligned}
\left\langle V\left(\widehat{u}_{0}\right),\left(u_{0}-\widehat{u}_{0}\right)^{+}\right\rangle & =\int_{\Omega} \widehat{h}_{+}\left(z, \widehat{u}_{0}\right)\left(u_{0}-\widehat{u}_{0}\right)^{+} d z \\
& =\int_{\Omega} f\left(z, u_{0}\right)\left(u_{0}-\widehat{u}_{0}\right)^{+} d z(\text { see }(3.24)) \text { and }(3.10) \\
& =\left\langle V\left(u_{0}\right),\left(u_{0}-\widehat{u}_{0}\right)^{+}\right\rangle
\end{aligned}
$$

hence

$$
\left\langle V\left(u_{0}\right)-V\left(\widehat{u}_{0}\right),\left(u_{0}-\widehat{u}_{0}\right)^{+}\right\rangle=0,
$$

therefore

$$
\int_{\left\{u_{0}>\widehat{u}_{0}\right\}}\left(a\left(z, D u_{0}\right)-a\left(z, D \widehat{u}_{0}\right), D u_{0}-D \widehat{u}_{0}\right)_{\mathbb{R}^{N}} d z=0
$$

and we conclude that

$$
\left|\left\{u_{0}>\widehat{u}_{0}\right\}\right|_{N}=0 \text {, i.e., } u_{0} \leq \widehat{u}_{0} .
$$

Similarly, acting on (3.25) with $\left(\widehat{u}_{0}-C_{+}\right)^{+} \in W_{0}^{1, p}(\Omega)$ and using hypothesis $\mathbf{H}_{1}(f)(i v)$, we show that $\widehat{u}_{0} \leq C_{+}$. Therefore

$$
\widehat{u}_{0} \in\left[u_{0}, C_{+}\right]=\left\{u \in W_{0}^{1, p}(\Omega): u_{0}(z) \leq u(z) \leq C_{+} \text {a.e. in } \Omega\right\}
$$

and so, (3.25) becomes

$$
V\left(\widehat{u}_{0}\right)=N_{f}\left(\widehat{u}_{0}\right)(\text { see }(3.24)) \text { and }(3.10)
$$

hence

$$
-\operatorname{div} a\left(z, D \widehat{u}_{0}(z)\right)=f\left(z, \widehat{u}_{0}(z)\right) \text { for a.e. } z \in \Omega,\left.\widehat{u}_{0}\right|_{\partial \Omega}=0
$$

and, by nonlinear regularity, it follows that $\widehat{u}_{0} \in$ int $\mathcal{C}_{+}$solves (1.1).

If $\widehat{u}_{0} \neq u_{0}$, then this is the desired second positive smooth solution of (1.1) such that $u_{0} \leq \widehat{u}_{0}, u_{0} \neq \widehat{u}_{0}$, so we are done.

Now we assume that $\widehat{u}_{0}=u_{0}$. Recall that $u_{0}(z)<C_{+}$for all $z \in \bar{\Omega}$ (see Proposition 3). We have

$$
\left.\psi_{+}\right|_{\left[0, C_{+}\right]}=\left.\widehat{\psi}_{+}\right|_{\left[0, C_{+}\right]}(\operatorname{see}(3.24) \text { and }(3.10)) \text {. }
$$


Therefore $\widehat{u}_{0}=u_{0}$ is a local $C_{0}^{1}(\bar{\Omega})$-minimizer of $\psi_{+}$, hence it is also a $W_{0}^{1, p}(\Omega)$-minimizer of $\psi_{+}$(see Proposition 2). This proves Claim (2).

Since by Claim (2), $u_{0}$ is a local minimizer of $\psi_{+}$, we may assume that it is an isolated critical point of $\psi_{+}$. Otherwise, we can find $\left\{u_{n}\right\}_{n \geq 1} \subseteq W_{0}^{1, p}(\Omega)$ such that

$$
u_{n} \rightarrow u_{0} \text { in } W_{0}^{1, p}(\Omega) \text { and } \psi_{+}^{\prime}\left(u_{n}\right)=0 \text { for all } n \geq 1 .
$$

As above, we can show that $u_{n} \geq u_{0}$. This, (3.10) and nonlinear regularity imply that $\left\{u_{n}\right\}_{n \geq 1} \subseteq$ int $\mathcal{C}_{+}$is a sequence of distinct positive smooth solutions of (1.1), and so we are done.

Therefore, we may consider that $u_{0}$ is an isolated critical point of $\psi_{+}$, and as in Aizicovici et al. [2] (see the proof of Proposition 29), we can find $r \in(0,1)$ small, such that

$$
\psi_{+}\left(u_{0}\right)<\inf \left\{\psi_{+}(u):\left\|u-u_{0}\right\|=r\right\}=: \eta_{r}^{+} .
$$

By virtue of hypothesis $\mathbf{H}_{1}(f)(i i)$

$$
\psi_{+}\left(t \widehat{u}_{1}\right) \rightarrow-\infty \quad \text { as } n \rightarrow \infty
$$

Then, Claim 1 together with (3.26) and (3.27) enables us to use Theorem 1 (the mountain pass theorem), and so we can find $\widehat{u} \in W_{0}^{1, p}(\Omega)$ such that

$$
\psi_{+}\left(u_{0}\right)<\eta_{r}^{+} \leq \psi_{+}(\widehat{u})
$$

and

$$
\psi_{+}^{\prime}(\widehat{u})=0
$$

From (3.28) it follows that

$$
\widehat{u} \neq u_{0} \text {. }
$$

From (3.29) we deduce that $u_{0} \leq \widehat{u}$ and that $\widehat{u} \in$ int $\mathcal{C}_{+}$(nonlinear regularity) solves problem (1.1). (See the argument following (3.25)).

Similarly, working on the negative half-axis and using $v_{0} \in-$ int $\mathcal{C}_{+}$ (see Proposition 3), we generate a second negative smooth solution $\widehat{v} \in$ $-\operatorname{int} \mathcal{C}_{+}, \widehat{v} \neq v_{0}, \widehat{v} \leq v_{0}$.

\section{A multiplicity theorem}

In this section we produce a fifth nontrivial smooth solution of (1.1) and state a complete multiplicity result for this problem. To do this, we need to strenghten the hypothesis on $f(z,$.$) near zero. The new hypotheses on f(z,$.$) are the$ following:

$\mathbf{H}_{2}(f): f: \Omega \times \mathbb{R} \rightarrow \mathbb{R}$ is a Carathéodory function such that $f(z, 0)=0$ for almost all $z \in \Omega$, hypotheses

$(i),(i i),(i v),(v)$ are the same as the corresponding hypotheses $\mathbf{H}_{1}(f)(i),(i i),(i v),(v)$ and 
(iii) there exists $\widehat{\eta} \geq \frac{C_{1}}{p-1} \widehat{\lambda}_{2}$ such that

$$
\widehat{\eta} \leq \liminf _{x \rightarrow 0} \frac{f(z, x)}{|x|^{p-2} x} \text { uniformly for a.e. } z \in \Omega .
$$

Now we can state and prove the full multiplicity theorem for problem (1.1).

Theorem 2. If hypotheses $\mathbf{H}(\mathbf{a})$ and $\mathbf{H}_{2}(f)$ hold, then problem (1.1) has at least five nontrivial smooth solutions

$$
\begin{aligned}
& u_{0}, \widehat{u} \in \operatorname{int} \mathcal{C}_{+}, u_{0} \leq \widehat{u}, u_{0} \neq \widehat{u} \\
& \widehat{v}, v_{0} \in-i n t \mathcal{C}_{+}, \widehat{v} \leq v_{0}, v_{0} \neq \widehat{v}
\end{aligned}
$$

and

$$
y_{0} \in C_{0}^{1}(\bar{\Omega}) \backslash\{0\} .
$$

Proof. The existence of the nontrivial constant sign solutions $u_{0}, \widehat{u} \in$ int $\mathcal{C}_{+}$ and $\widehat{v}, v_{0} \in-$ int $\mathcal{C}_{+}$such that $u_{0} \leq \widehat{u}, u_{0} \neq \widehat{u}, \widehat{v} \leq v_{0}, v_{0} \neq \widehat{v}$ follows from Propositions 3 and 4 . So, we need to produce the fifth nontrivial smooth solution. Evidently we may assume that $\left\{u_{0}, \widehat{u}, v_{0}, \widehat{v}\right\}$ are all the nontrivial constant sign solutions (otherwise, we already have a fifth nontrivial solution). Let

$$
\rho=\max \left\{\left\|u_{0}\right\|_{\infty},\left\|v_{0}\right\|_{\infty}\right\}
$$

and let $\xi_{\rho}>0$ as postulated by hypothesis $\mathbf{H}_{2}(f)(v)$. We introduce the following truncation of $f(z,$.$) :$

$$
\tilde{f}(z, x)= \begin{cases}f\left(z, v_{0}(z)\right)+\xi_{\rho}\left|v_{0}(z)\right|^{p-2} v_{0}(z) & \text { if } x<v_{0}(z) \\ f(z, x)+\xi_{\rho}|x|^{p-2} x & \text { if } v_{0}(z) \leq x \leq u_{0}(z) \\ f\left(z, u_{0}(z)\right)+\xi_{\rho} u_{0}(z)^{p-1} & \text { if } u_{0}(z)<x\end{cases}
$$

This is a Carathéodory function. We set

$$
\widetilde{F}(z, x)=\int_{0}^{x} \tilde{f}(z, s) d s
$$

and consider the $C^{1}$-functional $\widetilde{\varphi}: W_{0}^{1, p}(\Omega) \rightarrow \mathbb{R}$ defined by

$$
\widetilde{\varphi}(u)=\int_{\Omega} G(z, D u) d z+\frac{\xi_{\rho}}{p}\|u\|_{p}^{p}-\int_{\Omega} \widetilde{F}(z, u) d z \quad \text { for all } u \in W_{0}^{1, p}(\Omega) .
$$

Claim: $u_{0}$ and $v_{0}$ are both local minimizers of $\tilde{\varphi}$.

Let

$$
\widetilde{f}_{+}(z, x)=\tilde{f}\left(z, x^{+}\right), \widetilde{F}_{+}(z, x)=\int_{0}^{x} \widetilde{f}_{+}(z, s) d s
$$

and

$\widetilde{\varphi}_{+}(u)=\int_{\Omega} G(z, D u) d z+\frac{\xi_{\rho}}{p}\|u\|_{p}^{p}-\int_{\Omega} \widetilde{F}_{+}(z, u) d z \quad$ for all $u \in W_{0}^{1, p}(\Omega)$. 
Then $\widetilde{\varphi}_{+} \in C^{1}\left(W_{0}^{1, p}(\Omega)\right)$ is coercive and sequentially weakly lower semicontinuous. So, we can find $\widetilde{u}_{0} \in W_{0}^{1, p}(\Omega)$ such that

$$
\widetilde{\varphi}_{+}\left(\widetilde{u}_{0}\right)=\inf \left\{\widetilde{\varphi}_{+}(u): u \in W_{0}^{1, p}(\Omega)\right\} .
$$

Then

$\widetilde{\varphi}_{+}^{\prime}\left(\widetilde{u}_{0}\right)=V\left(\widetilde{u}_{0}\right)-N_{\widetilde{f}_{+}}\left(u_{0}\right)=0$ where $N_{\widetilde{f}_{+}}(u)()=.\widetilde{f}_{+}(., u()$.$) \quad for all u \in W_{0}^{1, p}(\Omega)$.

Hence

$$
\widetilde{u}_{0} \in\left[0, u_{0}\right] \backslash\{0\}
$$

(as before, using $\mathbf{H}_{2}(f)$ (iii); see the proof of Proposition 3), therefore $\widetilde{u}_{0} \in$ int $\mathcal{C}_{+}$(cf. (3.4) and (3.5) is a nontrivial positive smooth solution of (1.1).

But recall that $\left\{u_{0}, \widehat{u}\right\}$ are the only nontrivial positive smooth solutions of (1.1). Since $u_{0} \leq \widehat{u}$, we have $\widetilde{u}_{0}=u_{0} \in \operatorname{int} \mathcal{C}_{+}$. Note that

$$
\left.\widetilde{\varphi}\right|_{\left[0, u_{0}\right]}=\left.\widetilde{\varphi}_{+}\right|_{\left[0, u_{0}\right]}
$$

and so it follows that $u_{0}$ is a local $C_{0}^{1}(\bar{\Omega})$-minimizer of $\widetilde{\varphi}$, hence, by virtue of Proposition 2 , it is also a $W_{0}^{1, p}(\Omega)$-minimizer of $\widetilde{\varphi}$.

A similar argument works for $v_{0} \in-i n t \mathcal{C}_{+}$, using $\tilde{f}_{-}(z, x)=\tilde{f}\left(z,-x^{-}\right)$. This proves the Claim.

As before, we can check that the critical set $K_{\widetilde{\varphi}}$ (i.e., the set of all critical points) of $\widetilde{\varphi}$ is located in the order interval

$$
\left[v_{0}, u_{0}\right]=\left\{u \in W_{0}^{1, p}(\Omega): v_{0}(z) \leq u(z) \leq u_{0}(z) \text { a.e. in } \Omega\right\} .
$$

Without any loss of generality we may assume that

$$
\widetilde{\varphi}\left(v_{0}\right) \leq \widetilde{\varphi}\left(u_{0}\right)
$$

and that $u_{0}$ is an isolated critical point of $\widetilde{\varphi}$. So we can find $r \in(0,1)$ small such that

$$
\widetilde{\varphi}\left(v_{0}\right) \leq \widetilde{\varphi}\left(u_{0}\right)<\inf \left\{\widetilde{\varphi}(u):\left\|u-u_{0}\right\|=r\right\}=: \widetilde{\eta}_{r} .
$$

Since $\widetilde{\varphi}$ is coercive (see (4.1)), it satisfies the PS-condition. This fact together with (4.2) allows the use of Theorem 1 (the mountain pass theorem). So, we can find $y_{0} \in W_{0}^{1, p}(\Omega)$ such that

$$
\widetilde{\varphi}\left(v_{0}\right) \leq \widetilde{\varphi}\left(u_{0}\right)<\widetilde{\eta}_{r} \leq \widetilde{\varphi}\left(y_{0}\right)
$$

and

$$
\widetilde{\varphi}^{\prime}\left(y_{0}\right)=0 .
$$

From (4.3) we see that $y_{0} \notin\left\{v_{0}, u_{0}\right\}$. From (4.1) it follows that $y_{0} \in\left[v_{0}, u_{0}\right] \cap$ $C_{0}^{1}(\bar{\Omega})$ (nonlinear regularity) and so $y_{0}$ is a smooth solution of problem (1.1) $($ see $(4.1))$. It remains to show that $y_{0} \neq 0$. From Theorem 1 , we know that

$$
\widetilde{\varphi}\left(v_{0}\right)=\inf _{\gamma \in \Gamma} \max _{t \in[0,1]} \widetilde{\varphi}(\gamma(t)),
$$

where

$$
\Gamma=\left\{\gamma \in C\left([0,1], W_{0}^{1, p}(\Omega)\right): \gamma(0)=v_{0}, \gamma(1)=u_{0}\right\}
$$


According to (4.5), if we can find $\gamma_{*} \in \Gamma$ such that

$$
\left.\widetilde{\varphi}\right|_{\gamma_{*}}<0=\widetilde{\varphi}(0) \text {, }
$$

then $y_{0} \neq 0$. To this end let $M=W_{0}^{1, p}(\Omega) \cap \partial B_{1}^{L^{p}}$ furnished with the $W_{0}^{1, p}(\Omega)$ topology and $M_{c}=C_{0}^{1}(\bar{\Omega}) \cap M$ equipped with the $C_{0}^{1}(\bar{\Omega})$ topology. The set $M_{c}$ is dense in $M$ for the $W_{0}^{1, p}(\Omega)$-topology. We consider the following spaces of paths:

$$
\Gamma_{0}=\left\{\gamma_{0} \in C([-1,1], M): \gamma_{0}(-1)=-\widehat{u}_{1}, \gamma_{0}(1)=\widehat{u}_{1}\right\}
$$

and

$$
\Gamma_{0}^{c}=\left\{\gamma \in C\left([-1,1], M_{c}\right): \gamma(-1)=-\widehat{u}_{1}, \gamma(1)=\widehat{u}_{1}\right\} .
$$

Evidently $\Gamma_{0}^{c}$ is dense in $\Gamma_{0}$ for the $C([-1,1], M)$ topology. By virtue of hypothesis $\mathbf{H}_{2}(f)($ iii $)$, we can find $\widehat{\eta}_{1} \in\left(\frac{C_{1}}{p-1} \widehat{\lambda}_{2}, \widehat{\eta}\right)$ and $\delta_{0}>0$ such that

$$
\frac{f(z, x)}{|x|^{p-2} x} \geq \widehat{\eta}_{1} \quad \text { for a.e. } z \in \Omega \text {, all } 0<|x| \leq \delta_{0},
$$

hence

$$
F(z, x) \geq \frac{\widehat{\eta}_{1}}{p}|x|^{p} \quad \text { for a.e. } z \in \Omega \text {, all }|x| \leq \delta_{0} .
$$

From the density of $\Gamma_{0}^{c}$ in $\Gamma_{0}$ and the minimax characterization of $\widehat{\lambda}_{2}$ (see Sect. 2) we see that we can find $\gamma_{0} \in \Gamma_{0}^{c}$ such that

$$
\left\|D \gamma_{0}(t)\right\|_{p}^{p} \leq \widehat{\lambda}_{2}+\varepsilon \quad \text { for all } \mathrm{t} \in[-1,1] \text {, with } \frac{C_{1}}{p-1} \varepsilon \in\left(0, \widehat{\eta}_{1}-\frac{C_{1}}{p-1} \widehat{\lambda}_{2}\right) \text {. }
$$

We note that the set $\gamma_{0}([-1,1])$ is compact in $C_{0}^{1}(\bar{\Omega})$ and recall that $u_{0} \in$ int $\mathcal{C}_{+}$and $v_{0} \in-$ int $\mathcal{C}_{+}$. So, we can find $\widehat{\xi} \in(0,1)$ small enough, such that $|\widehat{\xi} u(z)| \leq \delta_{0} \quad$ for all $z \in \bar{\Omega}$ and $\widehat{\xi} u \in\left[v_{0}, u_{0}\right], \quad$ both for all $u \in \gamma_{0}([-1,1])$.

Then for all $u \in \gamma_{0}([-1,1])$, we have

$$
\begin{aligned}
& \widetilde{\varphi}(\widehat{\xi} u)=\int_{\Omega} G(z, \widehat{\xi} D u) d z-\int_{\Omega} F(z, \widehat{\xi} u) d z(\text { see }(4.1) \text { and }(4.8)) \\
& \quad \leq \frac{C_{1} \widehat{\xi}^{p}}{p(p-1)}\|D u\|_{p}^{p}-\int_{\Omega} F(z, \widehat{\xi} u) d z(\text { see Corollary } 1) \\
& \quad \leq \frac{C_{1} \widehat{\xi}^{p}}{p(p-1)}\left(\widehat{\lambda}_{2}+\varepsilon\right)-\frac{\widehat{\xi}^{p}}{p} \widehat{\eta}_{1}\left(\text { see }(4.6),(4.7),(4.8) \text { and recall that }\|u\|_{p}=1\right) \\
& \quad=\frac{\widehat{\xi}^{p}}{p}\left[\frac{C_{1}}{p-1}\left(\widehat{\lambda}_{2}+\varepsilon\right)-\widehat{\eta}_{1}\right]<0(\text { recall the choice of }) \varepsilon, \text { see }(4.7)
\end{aligned}
$$

So, if we set $\widehat{\gamma}_{0}=\widehat{\xi} \gamma_{0}$, then from (4.9) it follows that

$$
\left.\widetilde{\varphi}\right|_{\widehat{\gamma}_{0}}<0 .
$$

The critical set of $\widetilde{\varphi}_{+}$is $\left\{0, u_{0}\right\}$. Remark that

$$
\widetilde{\varphi}_{+}\left(u_{0}\right)=\inf \widetilde{\varphi}_{+}<0=\widetilde{\varphi}_{+}(0) \text {. }
$$


Invoking the second deformation theorem (see, Gasinski and Papageorgiou [16], p. 628) we can find a homotopy $h:[0,1] \times\left(\widetilde{\varphi}_{+}^{0} \backslash\{0\}\right) \rightarrow \widetilde{\varphi}_{+}^{0}$ such that

$$
h\left(1, \widetilde{\varphi}_{+}^{0} \backslash\{0\}\right)=\left\{u_{0}\right\}
$$

and

$$
\widetilde{\varphi}_{+}(h(t, u)) \leq \widetilde{\varphi}_{+}(u) \quad \text { for all } u \in \widetilde{\varphi}_{+}^{0} \backslash\{0\} \text { and all } t \in[0,1] .
$$

(Here $\left.\widetilde{\varphi}_{+}^{0}=\left\{u \in W_{0}^{1, p}(\Omega): \widetilde{\varphi}_{+}^{0}(u) \leq 0\right\}\right)$. We set

$$
\gamma_{0}^{+}(t)=h\left(t, \widehat{\xi} \widehat{u}_{1}\right) \quad \text { for all } t \in[0,1]
$$

Then

$$
\gamma_{0}^{+}(0)=h\left(0, \widehat{\xi} \widehat{u}_{1}\right)=\widehat{\xi} \widehat{u}_{1} \text { and } \gamma_{0}^{+}(1)=h\left(1, \widehat{\xi} \widehat{u}_{1}\right)=u_{0}
$$

(see $(4.11))$. So, $\gamma_{0}^{+}$is a continuous path connecting $\widehat{\xi} \widehat{u}_{1}$ and $u_{0}$. Moreover, from (4.10) and (4.12) it follows that

$$
\left.\widetilde{\varphi}_{+}\right|_{\gamma_{0}^{+}}<0 \text {. }
$$

For every $u \in \gamma_{0}^{+}([0,1])$, we have

$$
\begin{aligned}
\widetilde{\varphi}(u) & =\int_{\Omega} G(z, D u) d z+\frac{\xi_{\rho}}{p}\|u\|_{p}^{p}-\int_{\Omega}\left(\widetilde{F}\left(z, u^{+}\right)+\widetilde{F}\left(z,-u^{-}\right)\right) d z \\
& =\widetilde{\varphi}_{+}\left(u^{+}\right)-\int_{\Omega} \widetilde{F}\left(z,-u^{-}\right) d z
\end{aligned}
$$

By virtue of hypothesis $\mathbf{H}_{2}(f)(v)$, we see that $x=0$ is a global minimizer of the function

$$
x \rightarrow F(z, x)+\frac{\xi_{\rho}}{p}|x|^{p} \text { for } x \in[-\rho, \rho] .
$$

So, from (4.1) it follows that

$$
-\int_{\Omega} \widetilde{F}\left(z,-u^{-}\right) d z \leq 0
$$

Hence

$$
\widetilde{\varphi}(u) \leq \widetilde{\varphi}_{+}\left(u^{+}\right)(\operatorname{see}(4))
$$

therefore

$$
\left.\widetilde{\varphi}\right|_{\gamma_{0}^{+}}<0(\operatorname{see}(4.13))
$$

In a similar fashion, we produce a continuous path $\gamma_{0}^{-}$which connects $-\widehat{\xi} \widehat{u}_{1}$ and $v_{0}$ and such that

$$
\left.\widetilde{\varphi}\right|_{\gamma_{0}^{-}}<0
$$

We concatenate $\gamma_{0}^{-}, \gamma_{0}, \gamma_{0}^{+}$to obtain a path $\gamma_{*} \in \Gamma$ such that

$$
\left.\widetilde{\varphi}\right|_{\gamma_{*}}<0 \text { see }(4.10),(4.15)(4.16)
$$

and conclude that $y_{0} \neq 0$. 
Remarks. It is an interesting open question whether $y_{0} \in C_{0}^{1}(\bar{\Omega}) \backslash\{0\}$ is nodal. The lack of homogeneity of the differential operator creates serious technical difficulties which make the implementation of the strategy employed in [28] (Dirichlet problem) and [3], [1] (Neumann problems) problematic. In fact, we are not aware of any paper producing nodal solutions for equations driven by nonhomogeneous differential operators. All such results in the literature deal with the $p$-Laplacian. Again, if $a$ is $z$-independent (i.e., $a(z, y)=a(y)$ ), hypothesis $\mathbf{H}_{2}(f)(i v)$ can be replaced by $\mathbf{H}_{1}(f)(i v)^{\prime}$. (See the Remark following the proof of Proposition 3.)

\section{Acknowledgments}

The authors whish to thank the referee for his/her corrections and remarks.

\section{References}

[1] Aizicovici, S., Papageorgiou, N.S., Staicu, V.: Multiple solutions for super-linear p-Laplacian Neumann problems. Osaka J. Math. (2012) (to appear)

[2] Aizicovici, S., Papageorgiou, N.S., Staicu, V.: Degree theory for operators of monotone type and nonlinear elliptic equations with inequality constraints. Mem. Am. Math. Soc. 196. (2008)

[3] Aizicovici, S., Papageorgiou, N.S., Staicu, V.: Existence of multiple solutions with precise sign information for superlinear Neumann problems. Ann. Mat. Pura Appl. 188, 679-719 (2009)

[4] Aizicovici, S., Papageorgiou, N.S., Staicu, V.: On a p-superlinear Neumann p-Laplacian equation. Topol. Methods Nonlinear Anal. 34, 111-130 (2009)

[5] Arcoya, D., Ruiz, D.: The Ambrosetti-Prodi problem for the p-Laplace operator. Comm. Partial Diff. Equ. 31, 849-865 (2006)

[6] Brézis, H., Nirenberg, L.: $H^{1}$ versus $C^{1}$ local minimizers. C. R. Math. Acad. Sci. Paris 317, 465-472 (1993)

[7] Costa, D.G., Magalhaes, C.A.: Existence results for perturbations of the pLaplacian. Nonlinear Anal. 24, 409-418 (1995)

[8] Cuesta, M., deFigueiredo, D., Gossez, J.P.: The beginning of the Fucik spectrum for the p-Laplacian. J. Differ. Equ. 159, 212-238 (2002)

[9] Dabuleanu, S., Radulescu, V.: Multivalued boundary value problems involving Leray-Lions operators and discontinuous monlinearities. Rend. Circ. Mat. Palermo 152, 57-69 (2003)

[10] Damascelli, L.: Comparison theorems for some quasilinear degenerate elliptic operators and applications to symmetry and monotonicity results. Ann. Inst. H. Poincare Anal. Nonlineaire 15, 493-516 (1998) 
[11] DeNapoli, P., Mariani, M.C.: Mountain pass solutions to equations of p-Laplacian type. Nonlinear Anal. 54, 1205-1219 (2003)

[12] Duc, D.M., Vu, N.T.: Nonuniformly elliptic equations of p-Laplacian type. Nonlinear Anal. 61, 1483-1495 (2005)

[13] Fang, F., Liu, S.: Nontrivial solutions of superlinear p-Laplacian equations. J. Math. Anal. Appl. 351, 138-146 (2009)

[14] Fei, G.: On periodic solutions of superquadratic Hamiltonian systems. Electron. J. Diff. Equ. 2002, 1-12 (2002)

[15] Garcia Azorero, J., Manfredi, J., Peral Alonso, I.: Sobolev versus Holder local minimizers and global multiplicity for some quasilinear elliptic equations. Commun. Contemp. Math. 2, 385-404 (2000)

[16] Gasinski, L., Papageorgiou, N.S.: Nonlinear Analysis. Chapman \& Hall, Boca Raton (2006)

[17] Guo, Z., Zhang, Z.: $W^{1, p}$ versus $C^{1}$ local minimizers and multiplicity results for quasilinear elliptic equations. J. Math. Anal. Appl. 286, 32-50 (2003)

[18] Hu, S., Papageorgiou, N.S.: Positive solutions for nonlinear hemivariational inequalities. J. Math. Anal. Appl. 310, 161-176 (2005)

[19] Ioffe, A., Tichomirov, V: Theory of Extremal Problems. North Holland, Amster$\operatorname{dam}(1979)$

[20] Jeanjean, L.: On the existence of bounded Palais-Smale sequences and applications to a Landesman-Lazer-type problem set in $\mathbb{R}^{n}$. Proc. R. Soc. Edinburgh. Sect. A 129, 767-809 (1999)

[21] Kourogenis, N., Papageorgiou, N.S.: Nonlinear hemivariational inequalities of second order using the method of upper-lower solutions. Proc. Am. Math. Soc. 131, 2359-2369 (2003)

[22] Krystaly, A., Lisei, H., Varga, C.: Multiple solutions for p-Laplacian type equations. Nonlinear Anal. 68, 1375-1381 (2008)

[23] Kyritsi, S., O’Regan, D., Papageorgiou, N.S.: Existence of multiple solutions for nonlinear Dirichlet problems with nonhomogeneous differential operator. Adv. Nonlinear Stud. 10, 631-658 (2010)

[24] Ladyzhenskaya, O., Uraltseva, N.: Linear and Quasilinear Elliptic Equations. Academic Press, New York (1968)

[25] Li, C., Yang, C.: The existence of a nontrivial solution to a nonlinear boundary value problem of p-Laplacian type without the Ambrosetti-Rabinowitz condition. Nonlinear Anal. 72, 4607-4613 (2010)

[26] Lieberman, G.: Boundary regularity for solutions of degenerate elliptic equations. Nonlinear Anal. 12, 1203-1219 (1988)

[27] Miyagaki, O., Souto, M.: Superlinear problems without Ambrosetti and Rabinowitz growth condition. J. Differ. Equ. 245, 3628-3638 (2008) 
[28] Papageorgiou, N.S., Rocha, E.M.: On nonlinear parametric problems for p-Laplacian-like operators. Rev. R. Acad. Cienc. Exactas Fis. Nat. Ser. A. Mat. RACSAM 103, 177-200 (2009)

[29] Papageorgiou, N.S., Rocha, E.M., Staicu, V.: A multiplicity theorem for hemivariational inequalities with a p-Laplacian-like differential operator. Nonlinear Anal. 69, 1150-1163 (2008)

[30] Pucci, P., Serrin, J.: The Maximum Principle. Birkhauser, Basel (2007)

[31] Schechter, M., Zou, W.: Superlinear problems. Pacific J. Math 214, 145-160 (2004)

[32] Su, M.: Multiple solutions of a superlinear p-Laplacian equation without AR-condition. Appl. Anal. 89, 325-336 (2010)

[33] Tolksdorf, P.: On the Dirichlet problem for quasi-linear equations in domains with conical boundary points. Comm. Partial Differ. Equ. 8, 773-817 (1983)

[34] Vazquez, J.: A strong maximum principle for some quasilinear elliptic equations. Appl. Math. Optim. 12, 191-202 (1984)

[35] Zhang, Q.: A strong maximum principle for differential equations with nonstandard p(x)-growth conditions. J. Math. Anal. Appl. 312, 24-32 (2005)

Sergiu Aizicovici

Department of Mathematics

Ohio University

Athens

OH 45701

USA

e-mail: aizicovs@ohio.edu

Nikolaos S. Papageorgiou

Department of Mathematics

National Technical University

Zografou Campus

Athens 15780

Greece

e-mail: npapg@math.ntua.gr

Vasile Staicu

CIDMA, Department of Mathematics

University of Aveiro

Campus Universitário de Santiago

3810-193 Aveiro

Portugal

e-mail: vasile@ua.pt

Received: 10 October 2011.

Accepted: 14 July 2012. 